\title{
Targeting GLI factors to inhibit the Hedgehog pathway
}

\author{
Paola Infante ${ }^{1}$, Romina Alfonsi ${ }^{2}$, Bruno Botta ${ }^{3}$, Mattia Mori ${ }^{1}$, and \\ Lucia Di Marcotullio ${ }^{1,2,4}$
}

\author{
${ }^{1}$ Center for Life Nano Science@Sapienza, Istituto Italiano di Tecnologia, 00161 Rome, Italy \\ 2 Department of Molecular Medicine, “La Sapienza" University, 00161 Rome, Italy \\ ${ }^{3}$ Dipartimento di Chimica e Tecnologie del Farmaco, "La Sapienza" University, 00161 Rome, Italy \\ ${ }^{4}$ Pasteur Institute/Cenci Bolognetti Foundation, Rome, Italy
}

\begin{abstract}
Hedgehog $(\mathrm{Hh})$ signaling has emerged in recent years as an attractive target for anticancer therapy because its aberrant activation is implicated in several cancers. Major progress has been made in the development of SMOOTHENED (SMO) antagonists, although they have shown several limitations due to downstream SMO pathway activation or the occurrence of drug-resistant SMO mutations. Recently, particular interest has been elicited by the identification of molecules able to hit glioma-associated oncogene (GLI) factors, the final effectors of the Hh pathway, which provide a valid tool to overcome anti-SMO resistance. Here, we review results achieved in developing GLI antagonists, explaining their mechanisms of action and highlighting their therapeutic potential. We also underline the relevance of structural details in their discovery and optimization.
\end{abstract}

\section{Hh pathway and cancer}

The evolutionarily conserved Hh pathway plays a crucial role in patterning and organogenesis during early development, in adult tissue maintenance and repairing functions [1]. The $\mathrm{Hh}$ signaling represents a complex transduction pathway orchestrated by several regulatory components and post-translational events. A simplified model of Hh signaling describes that in the absence of Hh ligand (Sonic, Indian and Desert Hh), the PATCHED receptor (PTCH) inhibits the class F G-protein-coupled receptor (GPCR) SMO. When PTCH is engaged by Hh, it relieves the inhibition of SMO and the signal is transduced to the downstream transcription factors GLI1, GLI2, and GLI3, which in turn regulate the expression of $\mathrm{Hh}$ target genes involved in key cellular processes, such as cell cycle, survival, migration, and metabolism [2]. Given the significant involvement of $\mathrm{Hh}$ signaling in the development of several districts including pancreas, kidney, lung, nervous system, and limb [3-5], its misregulation results in multiple birth and developmental defects [6,7]. Aberrant Hh pathway activation is responsible for the tumorigenesis of several disparate human cancers including

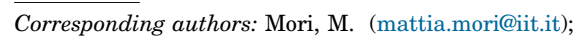

Di Marcotullio, L. (lucia.dimarcotullio@uniroma1.it).

Keywords: Hedgehog pathway; GLI antagonists; drug discovery; cancer.

0165-6147/

(C) 2015 Elsevier Ltd. All rights reserved. http://dx.doi.org/10.1016/j.tips.2015.05.006 medulloblastoma (MB), rhabdomyosarcoma, melanoma, basal cell carcinoma (BCC), and breast, lung, liver, stomach, prostate, and pancreas tumors [8-11]. Hh-dependent carcinogenesis may result from abnormal upregulation of Hh ligands or deregulation of the expression or function of downstream components such as loss of PTCH [12] or suppressor of fused SUFU (the main negative regulator of Hh signaling) [13], activating mutations of SMO [14], amplification or chromosomal translocation of GLI1 [15], GLI2 gene amplification, or stabilization of GLI2 protein. Moreover, alterations of phosphorylation, ubiquitylation, and acetylation post-translational processes can also contribute to Hh-dependent tumorigenesis by modulating GLI1 function [16-18]. Remarkably, Hh signaling is active in cancer stem cells (CSCs) of various tumor types $[19,20]$, sustaining the proliferation of these niche cells that are responsible for tumor relapse and resistance to conventional anticancer therapy. Indeed, the Hh pathway controls the functional properties of CSCs, such as selfrenewal, survival, metastatic spread, and neoangiogenesis by the regulation of stemness-determining genes such as Nanog, often overexpressed in cancer. Given the increasing evidences supporting the crucial role of the $\mathrm{Hh}$ pathway in cancer initiation, proliferation, metastasis, chemoresistance, and in the survival of CSCs $[10,17]$, its components represent attractive druggable targets for anticancer therapy.

\section{SMO antagonists: pitfalls and limitations}

The first Hh inhibitor discovered was cyclopamine, a naturally occurring alkaloid isolated from Veratrum californicum [21], which inactivates SMO by directly binding to its hepathelical bundle. Nevertheless, cyclopamine has shown several limitations as a drug candidate, such as toxicity and teratogenicity, poor oral bioavailability, and suboptimal pharmacokinetics with relatively short elimination half-life [22].

In recent years, drug discovery efforts directed against the Hh pathway have been focused predominantly on the development of SMO antagonists and a remarkable number of small molecules of natural, semisynthetic or synthetic origin have been developed and extensively reviewed in recent reports [23-25]. Several SMO antagonists have demonstrated efficacy in mouse xenografts and, most notably, have been investigated in clinical trials against a 
large range of metastatic and advanced cancers [26,27]. Among them, vismodegib (GDC-0449/Erivedge) became the first $\mathrm{Hh}$ inhibitor to receive approval from the USA FDA in January 2012 for the treatment of locally advanced or metastatic BCC [28].

Despite the initial enthusiasm, clinical development of SMO antagonists has ultimately proved disappointing, due to scarce pharmacokinetics, low selectivity on CSCs, severe side effects, and the emergence of drug resistance. Indeed, after an initial clinical response to treatment with vismodegib, a patient with metastatic MB showed tumor regrowth within 3 months due to $\mathrm{D} 473 \mathrm{H}$ point mutation that rendered SMO insensitive to the drug [29]. Furthermore, acquired resistance has been also observed in BCC patients under treatment with vismodegib and in a Phase I study of saridegib (IPI-926). In this study, nine patients with BCC, previously progressed on vismodegib, failed to respond to saridegib, suggesting the existence of overlapping mechanisms of resistance [30]. Recently, genomic analysis of SMO resistance to vismodegib in BCC patients has revealed a number of additional SMO mutations and variants that confer constitutive activity and drug resistance [31,32]. All variants have shown partial or complete resistance to vismodegib, while the aPKC-t/ $\lambda /$ GLI inhibitor PSI and the GLI2 antagonist arsenic trioxide (ATO) were both able to suppress Hh pathway activation in the presence of any SMO variants [31].

Several lines of evidence also suggest that cancer cells can acquire resistance to SMO antagonists via SMO-independent hyperactivation of the powerful downstream GLI transcription factors, or mutations at different nodal points of the Hh pathway. Indeed, preclinical and clinical trials have shown that SMO drug resistance can be the consequence of (i) GLI2 amplification during vismodegib or sonidegib (LDE-225) treatment; (ii) upregulation of noncanonical and synergistic GLI signaling [e.g., phosphoinositide 3-kinase (PI3K) pathway, observed during sonidegib treatment]; and (iii) increase of the expression of ATPbinding cassette transporters (ABCs), such as P-glycoprotein (Pgp), which diminishes drug efficacy by increasing its cellular clearance [29,33-36].

Moreover, the onset and progression of some types of Hh-driven cancers are related to $\mathrm{Hh}$-pathway-activating mutations downstream of SMO, such as loss of SUFU or GLI1 amplification, thus rendering SMO antagonists ineffective in these scenarios.

Not least, studies investigating systemic treatments with SMO antagonists have revealed several side effects including dysgeusia, alopecia, fatigue, nausea, diarrhea, decreased appetite, hyponatremia, weight loss, and especially muscle cramping due to noncanonical SMO signaling (SMO-AMP-activated protein kinase axis) and $\mathrm{Ca}^{2+}$ influx [37,38].

Consequently, the development of $\mathrm{Hh}$ inhibitors that modulate targets acting downstream of SMO or independently by SMO, such as GLI, has recently emerged as a more promising therapeutic strategy for the treatment of Hh-dependent tumor. This approach would allow overcoming anti-SMO resistance and adverse effects, which are responsible for $>50 \%$ dropouts rates in SMO antagonists clinical trials.

\section{GLI factors: new attractive targets in Hh-dependent tumors}

GLI transcription factors are the final effectors of the $\mathrm{Hh}$ pathway and share common structural features, such as five highly conserved tandem zinc fingers (ZFs), a fairly conserved N-terminal domain, several potential protein kinase A (PKA) binding sites, and additional conserved regions at the $\mathrm{C}$ terminus. Nevertheless, GLI proteins exert different functions in vivo: GLI1 acts only as a transcriptional activator, whereas GLI2 and GLI3 can act both as transcriptional activators and as repressors, depending on the specific cell context and on the activation state of $\mathrm{Hh}$ signaling. In absence of upstream Hh signaling, some protein kinases [PKA, glycogen synthase kinase (GSK) $3 \beta$ and casein kinase (CK)1] phosphorylate GLI proteins, leading to ubiquitylation/proteosome-dependent GLI1 degradation or GLI2 and GLI3 proteolytic cleavage into repressor forms (GLI2R and GLI3R). These events are mediated by Cullin1/beta-transducin repeats-containing proteins $(\beta$-TrCP) E3 ubiquitin ligase complex [1,39]. In contrast, the activation of $\mathrm{Hh}$ signaling inhibits this processing, resulting in full-length GLI2 and GLI3, which have activator function (GLI3A and GLI2A) [38]. The balance between activator and repressor functions of GLI transcription factors determines the status of the Hh transcriptional program and consequently the behavior of responding cells.

The fine interplay among post-translational modifications and intersection with other pathways, such as PI3K-AKT, has a crucial role in the regulation of GLI activity and in the generation of their repressor forms [40-42]. In addition to $\beta$-TrCP, for example, other E3 ligases belonging to the really interesting new gene (RING) (Cullin3/speckle-type POZ protein (HIB-SPOP)) or homologous to E6-AP carboxy terminus (HECT) (ITCH) family have been identified to be responsible for Drosophila and mammalian Ci/GLI proteolysis through phosphorylation-independent mechanisms [43,44]. Furthermore, acetylation/ubiquitylation interplay, mediated by histone deacetylases (HDACs) and members of Cul3 adaptor proteins family potassium channel tetramerization domain, (KCTDs) (KCASH, KCTD containing Cullin3 Adaptor Suppressor of Hedgehog), has been recently discovered as a key transcriptional checkpoint of GLI function [45,46].

Albeit several aspects of GLI regulation remain unclear, it is evident the key role of GLI in embryogenesis and adult homeostasis. Indeed, GLI factors activate the expression of a number of genes involved in functions related to tissue development, such as cell proliferation and differentiation (e.g., CyclinD1 and D2,N-Myc, Wnts, PDGFR,IGF2, FoxM1, FoxA2, Nkx2.2, FoxF1, Myf5, HES1, and IGFBP3), survival (Bcl2), self-renewal and cell fate determination (Bmi1 and Nanog), angiogenesis (VEGF), epithelial-mesenchymal transition (Snail1, Sip1, Elk1, and Msx2) and tumor cell invasiveness (Osteopontin) in several tissues [19,47]. Remarkably, GLI factors control the expression of both GLI1 and PTCH1, thus forming a feedback loop enhancing or repressing Hh response. Given the broad spectrum of cellular events under GLI control, dysregulation of GLI genes could clearly lead to unfavorable developmental and pathological consequences, such as oncogenesis. GLI1 was indeed the first Hh pathway gene found to be amplified in several cancers, such as glioblastoma, glial tumors, prostate cancer, 
and a panel of brain tumors in a study that identified GLI1 expression as the only reliable marker of Hh pathway activity [15,48]. Moreover, the level of GLI1 transcript can be used to discriminate BCC from certain other skin tumors [49] and GLI deregulations have been revealed in $\mathrm{MB}$, the most common malignant pediatric brain tumor. The primary mediator of $\mathrm{Hh}$ signaling, GLI2, has been also identified as pleiotropic oncogene, whose upregulation induces genomic instability and a number of the acquired characteristics of tumor cells [50]. Interestingly, GLI2 is upregulated in a wide variety of human tumors such as melanoma [51], prostate cancer [52], BCC [53], and hepatocellular carcinoma [54], becoming potentially an attractive therapeutic target. Noteworthy, several reports have highlighted noncanonical mechanisms of GLI activation, in addition or independent of upstream $\mathrm{Hh}$ signaling $[26,47,55]$. For instance, GLI expression, stability, and/or transcriptional activity in normal and cancer cells may be positively modulated via the persistent stimulation of different growth factor cascades, such as EGF/EGFR (epidermal growth factor receptor), Wnt/ $\beta$-catenin, and the TGF- $\beta 1 /$ TGF- $\beta R$ (transforming growth factor $\beta$ receptor) system [56]. The aberrant crosstalk among Hh signaling and these pathways can induce tumor transformation. In this regard, K-RAS and TGF- $\beta$ were shown to regulate GLI1 expression in absence of SMO and they collaborate with $\mathrm{Hh}$ signaling to initiate pancreatic ductal adenocarcinoma (PDAC) development [57]. In contrast, EGFR signaling modulates $\mathrm{Hh} / \mathrm{GLI}$ target gene expression during keratinocytes transformation inducing activation of JUN/activator protein-1 (AP1), which cooperates with GLI1 and GLI2 [58]. Moreover, the interaction between $\mathrm{Hh}$ and plateletderived growth factor (PDGF) signaling has been shown in cultured murine fibroblasts, BCC cells, and CNS tumors [59]. Activation of the Hh/GLI signaling was also observed in Ewing sarcoma family tumors (ESFTs), where the oncogenic transcription factor Ewing's Sarcoma/Friend leukemia integration 1 (EWS/FL1), resulting from the chromosomal translocation $t(11 ; 22)$, induces directly GLI1 expression [60]. Furthermore, PI3K/AKT signaling was described to negatively regulate the degradation of GLI2 and potentiate GLI1 transcriptional activity [61]. Hh signaling is also differently modulated by distinct members of the PKC family: upregulation of $\mathrm{aPKC} / \mathrm{\lambda}$ potentiates $\mathrm{Hh}$ signaling by directly phosphorylating and activating GLI1 and because of $\mathrm{aPKC} / \mathrm{\lambda}$ is also an $\mathrm{Hh}$ target gene, it sustains a positive feedback loop contributing to Hh activation [62].

It is important to consider that, whatever alteration leads to aberrant Hh pathway activation, such as genetic mutations of pathway components or other SMO-dependent or -independent mechanisms, all trigger the downstream effector GLI1. For this reason, GLI factors are emerging as attractive targets for the development of novel anticancer drugs. However only a few GLI antagonists have been identified, most likely due to the lack of structural details related to GLI activity.

Most effective GLI antagonists developed so far are herein reviewed and classified based on their mechanism of action. Direct GLI antagonists act by contacting directly GLI effectors and blocking their transcriptional functions, whereas indirect GLI antagonists inhibit GLI functions through mechanisms that control GLI activity, such as proteolytic degradation or post-translational modifications.

\section{Indirect GLI antagonists}

\section{Cyclohexyl-methyl aminopyrimidines (CMAPs)}

A series of CMAPs (Figure 1) have been identified by a phenotypic screening approach, designed to classify compounds as SMO or non-SMO antagonists. Bassilana and coworkers have discovered a CMAP chemotype able to block GLI functions and, through a chemoproteomics strategy, they also have identified the orphan GPCR GPR39 as the specific target of this molecule [63]. Although the authors failed to demonstrate the direct binding of CMAP to GPR39, data document that CMAP is a specific agonist of this receptor. Indeed, ectopic expression or knockdown of GPR39 conferred or decreased, respectively, responsiveness to CMAP. Interestingly, CMAP analogs were able to inhibit GLI-activated signaling following overexpression of GLI1 or GLI2, providing evidence that CMAPs affect $\mathrm{Hh}$ signaling (Table 1). Specifically, CMAPs-activated GPR39 leads to inositol triphosphate production and activation of mitogen-activated protein kinase (MAPK) pathway, thus repressing GLI signaling. However, the mechanism by which GPR39 activation impinges GLI function has not been elucidated (Figure 2). Since MAPK activation occurs also via $\mathrm{G}_{\mathrm{i}}$-coupled signaling and $\beta$ arrestin recruitment, the discovery of CMAPs/GPR39 axis has identified a new mechanism for Hh inhibition downstream of SMO, triggering signaling pathways that cooperate to counteract $\mathrm{Hh}$ activation.

\section{JQ1 and I-BET151}

Recently, epigenetic enzymes have emerged as druggable targets for attenuating the growth of Hh-dependent tumors [64]. A new class of compounds targeting bromo and extra C-terminal (BET) bromodomain (BRD) proteins has been described to specifically affect GLI transcriptional activity. Members of the BET family proteins (BRD1-4) are involved in cell cycle progression, chromatin compaction, and chemoresistance. These proteins, through their bromodomains, bind to acetylated lysines in histones, and through their interaction with the positive transcription elongation factor ( $\mathrm{p}$-TEFb) stimulate RNA polymerase II activity and enhance gene expression [65]. Among different BET enzymes, suggestive evidences identify BRD4 as an attractive candidate target for developing therapeutics against Hh-driven tumors. Tang and colleagues have demonstrated that BRD4 activates Hh signaling in a ligand-independent manner, finely tuning GLI1- and GLI2-mediated transcription by direct occupancy of their promoters. Accordingly, they have identified the BET inhibitor JQ1 (Figures 1 and 2) as a potent GLI antagonist that alters the recruitment of GLI proteins on their target genes, determining growth arrest and cell death of tumors from patients with aberrant Hh pathway activation (Table 1). Importantly, JQ1 also impaired GLI signaling in a context of acquired resistance to SMO antagonism. Another BRD4 inhibitor, I-BET151 (Figures 1 and 2), has been recently described to suppress the expression of $\mathrm{Hh}$ target genes in a SMO-independent fashion, thus inhibiting MB cells growth, 


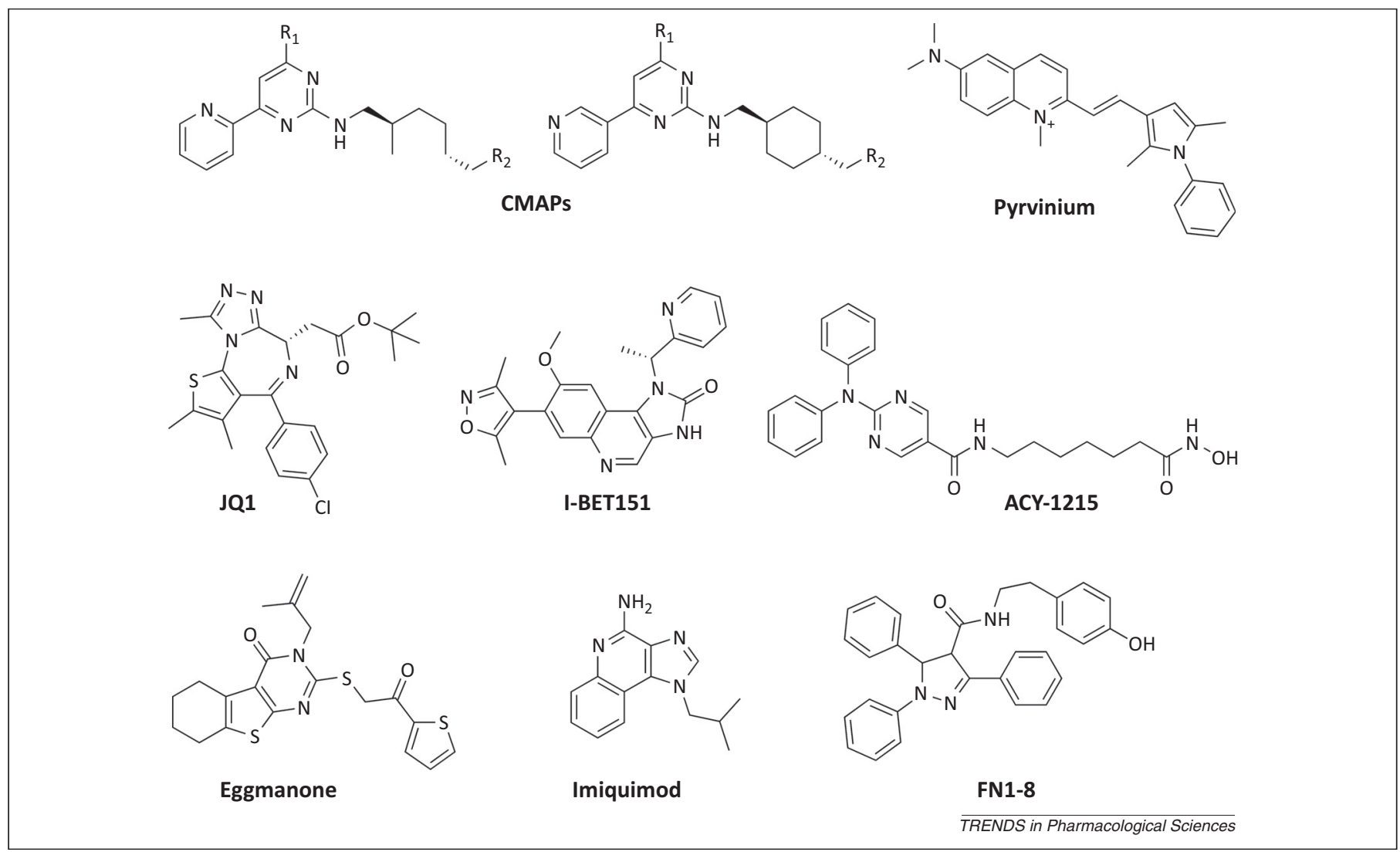

Figure 1. Indirect GLI antagonists. Chemical structure of the most effective GLI antagonists acting by an indirect mechanism of action. Abbreviations: CMAP, cyclohexylmethyl aminopyrimidines; GLI, glioma-associated oncogenes.

in vitro and in vivo (Table 1) [66]. Notably, BRD4 has been found overexpressed in PDAC cell lines, being able to sustain a high proliferative growth rate and chemoresistance [67]. All these finding suggest that BET inhibitors can represent an innovative therapeutic tool for the treatment of aggressive Hh-dependent tumors besides MB and BCC.

\section{HDAC inhibitors (HDACis)}

HDACs are a class of enzymes known as epigenetic readers that play a key role in the regulation of multiple biological events. Members of HDACs family are involved in the control of Hh pathway. GLI1 and GLI2 have been reported to be acetylated proteins $[45,68]$ and their HDAC-mediated deacetylation has been described to promote their transcriptional activation. This mechanism sustains a positive autoregulatory loop through $\mathrm{Hh}$-induced upregulation of HDAC1 and plays a role in ubiquitylation-dependent control of GLI1 function. Indeed, a Cul3 E3 ubiquitin ligase complex, formed by a family of the adaptor proteins KCASH1, KCASH2, and KCASH3, triggers HDAC1 degradation $[45,46]$. These findings unveil an integrated HDAC- and ubiquitin-mediated circuitry, where acetylation of GLI proteins functions as an unexpected key transcriptional checkpoint of $\mathrm{Hh}$ signaling. These data identify HDACis as Hh antagonists able to prevent transcriptional activity by inducing GLI1 and GLI2 hyperacetylation (Figure 2) $[41,45,68]$. Next to HDAC1, also HDAC5 and HDAC9 were found to be upregulated in MB, and their expression was associated with poor prognosis [69]. Interestingly, Dhanyamraju and colleagues have recently observed that another deacetylase, HDAC6, implicated in primary cilium biology, is overexpressed in a murine model of Hh-driven MB. These findings suggest that selective inhibition of HDAC6 can represents a new therapeutic approach in the treatment of $\mathrm{Hh}$-dependent malignancies. Indeed, the specific HDAC6 antagonist, ACY-1215 [70] (Figures 1 and 2 and Table 1), reduced strikingly in vivo tumor growth. Hence, HDAC6, despite exerting a dichotomous role (it is required to achieve full pathway activity, but it has also been described to repress basal Hh target gene transcription) is a critical player in Hh pathway regulation, promoting the maximum expression of a subset of GLI target genes [70]. Since the upregulation of HDACs has been documented in various types of tumors, the development of novel potent and selective HDACi is ongoing. In this regard, several chemical classes of HDACi are currently being tested in human cancer therapy. To date, three HDACi (vorinostat [SAHA], romidepsin and belinostat) have been approved by the FDA for treating refractory cutaneous and peripheral $\mathrm{T}$ cell lymphoma, while other HDACis have entered clinical trials in both solid and hematological malignancies [71,72]. More importantly, it has been reported that SAHA can penetrate the blood-brain barrier and induces apoptosis in mouse models of MB [73].

\section{Pyrvinium}

Recently, the anthelmintic drug pyrvinium (Figure 1) has been described to affect on Hh signaling by inducing GLI destabilization [74,75]. In particular, pyrvinium has been described as a specific activator of CK1 $\alpha$, a kinase that negatively regulates GLI transcription factors by promoting the processing of GLI3 into repressor form and GLI2 
Table 1. Efficacy and in vivo study of GLI antagonists

\begin{tabular}{|c|c|c|c|c|c|}
\hline Compound & Assay & Efficacy $^{a}$ & In vivo mouse study & Dosage & Refs \\
\hline CMAP-3 & TM3 (1 nM Ag1.5) Gliluc-assay & $0.004 \mu \mathrm{M}\left(\mathrm{EC}_{50}\right)$ & n.d. & n.d. & [63] \\
\hline CMAP-5 & TM3 (1 nM Ag1.5) Gliluc-assay & $0.02 \mu \mathrm{M}\left(\mathrm{EC}_{50}\right)$ & n.d. & n.d. & [63] \\
\hline CMAP-6 & TM3 (1 nM Ag1.5) Gliluc-assay & n.d. & n.d. & n.d. & [63] \\
\hline CMAP-7 & TM3 (1 nM Ag1.5) Gliluc-assay & $0.01 \mu \mathrm{M}\left(\mathrm{EC}_{50}\right)$ & n.d. & n.d. & [63] \\
\hline I-BET151 & $\begin{array}{l}\text { Shh-Light2 cells (SAG)/firefly } \\
\text { luciferase }\end{array}$ & $31 \mathrm{nM}\left(\mathrm{IC}_{50}\right)$ & $\begin{array}{l}\text { Allograft of spontaneous } \\
\text { MBs from } \mathrm{Ptch}^{+/-} \text {mice }\end{array}$ & $30 \mathrm{mg} / \mathrm{kg}$ & [66] \\
\hline JQ-1 & $\begin{array}{l}\text { Cell viability in Smo WT -MB and } \\
\text { Med1-MB cells }\end{array}$ & $\sim 50-150 \mathrm{nM}\left(\mathrm{IC}_{50}\right)$ & $\begin{array}{l}\text { Allograft of Med1-MB } \\
\text { cells; SmoWT-MB or } \\
\text { SmoD477G-MB cells. } \\
\text { Allograft model of Ptch }{ }^{+/} \text {; } \\
\text { K14-creER2; } p 53^{\text {flox/flox }} \\
\text { derived mouse BCC cells }\end{array}$ & $50 \mathrm{mg} / \mathrm{kg}$ & [109] \\
\hline ACY-1215 & $\begin{array}{l}\text { Shh-Light2 cells (SAG)/firefly } \\
\text { luciferase }\end{array}$ & n.d. & $\begin{array}{l}\text { Allograft of primary } \\
\text { SmoA1 MB cells (MB99-1 } \\
\text { cells) }\end{array}$ & $50 \mathrm{mg} / \mathrm{kg}$ & [70] \\
\hline Pyrvinium & $\begin{array}{l}\text { Shh-Light2cells (SAG or SHH)/ } \\
\text { firefly luciferase }\end{array}$ & $\sim 10 \mathrm{nM}\left(\mathrm{IC}_{50}\right)$ & $\begin{array}{l}\text { (a) Allograft of } \\
\text { spontaneous MBs from } \\
\text { Ptch }^{+-} \text {mice } \\
\text { (b) APC }\end{array}$ & $\begin{array}{l}\text { (a) } 0.8 \mathrm{mg} / \mathrm{kg} \\
5 \mathrm{mg} / \mathrm{kg} \\
\text { (b) } 25 \mathrm{mg} / \mathrm{kg}\end{array}$ & {$[74,75]$} \\
\hline GANT58 & $\begin{array}{l}\text { Shh-Light2 cells (SAG)/firefly } \\
\text { luciferase }\end{array}$ & $\sim 5 \mu \mathrm{M}\left(\mathrm{IC}_{50}\right)$ & $\begin{array}{l}\text { Xenograft of } 22 R v 1 \\
\text { prostate cancer cells }\end{array}$ & $50 \mathrm{mg} / \mathrm{kg}$ & [81] \\
\hline GANT61 & $\begin{array}{l}\text { Shh-Light2 cells (SAG)/firefly } \\
\text { luciferase }\end{array}$ & $\sim 5 \mu \mathrm{M}\left(\mathrm{IC}_{50}\right)$ & $\begin{array}{l}\text { Xenograft of } 22 \mathrm{Rv} 1 \\
\text { prostate cancer cells } \\
\text { Xenograft of Huh7 } \\
\text { hepatocellular carcinoma } \\
\text { cells } \\
\text { Orthotopic xenograft of } \\
\text { CFPAC-1 pancreatic } \\
\text { cancer cells }\end{array}$ & $50 \mathrm{mg} / \mathrm{kg}$ & {$[81,86,91]$} \\
\hline HPI-2 & $\begin{array}{l}\text { GLI1 overexpressing NIH 3T3 } \\
\text { cells/firefly luciferase }\end{array}$ & $>30 \mu \mathrm{M}\left(\mathrm{IC}_{50}\right)$ & - & - & [100] \\
\hline $\mathrm{HPI}-3$ & $\begin{array}{l}\text { GLI1 overexpressing NIH 3T3 } \\
\text { cells/firefly luciferase }\end{array}$ & $>30 \mu \mathrm{M}\left(\mathrm{IC}_{50}\right)$ & - & - & [100] \\
\hline $\mathrm{HPI}-4$ & $\begin{array}{l}\text { GLI1 overexpressing NIH } 3 \text { T3 } \\
\text { cells/firefly luciferase }\end{array}$ & $>30 \mu \mathrm{M}\left(\mathrm{IC}_{50}\right)$ & - & - & [100] \\
\hline ATO & NIH 3T3 (ShhN) Gliluc-assay & $0.7 \mu \mathrm{M}\left(I \mathrm{C}_{50}\right)$ & $\begin{array}{l}\text { (a) Allograft of primary } \\
\text { MBs from } \mathrm{Ptch}^{+/} \mathrm{p} 53^{-1-} \\
\text { mice } \\
\text { (b) Allograft of BCC tumor } \\
\text { derived from } \\
\text { Ptch }^{+/-} ; K 14-c r e E R 2 ; \\
p 53^{\text {flox flox }}\end{array}$ & $\begin{array}{l}\text { (a) } 2.5-10 \mathrm{mg} / \mathrm{kg} \\
\text { (b) } 7.5 \mathrm{mg} / \mathrm{kg}\end{array}$ & {$[92,94]$} \\
\hline GlaB & $\begin{array}{l}\text { GLI1-overespressing HEK293T } \\
\text { cells/firefly luciferase }\end{array}$ & $12 \mu \mathrm{M}\left(\mathrm{IC}_{50}\right)$ & $\begin{array}{l}\text { (a) Allograft of } \\
\text { spontaneous MBs from } \\
\text { Ptch }{ }^{+-} \text {mice } \\
\text { (b) Orthotopic xenograft of } \\
\text { Daoy MB cells } \\
\text { (c) Allograft of ASZ001 } \\
\text { BCC cells }\end{array}$ & $\begin{array}{l}\text { (a)-(b) } \\
35 \mathrm{mg} / \mathrm{kg} \\
\text { (c) } 50 \mathrm{mg} / \mathrm{kg}\end{array}$ & [106] \\
\hline
\end{tabular}

${ }^{a}$ Efficacy is expressed as $\mathrm{EC}_{50}$ or $\mathrm{IC}_{50}$ as reported in parentheses. n.d., not determined/unknown. 


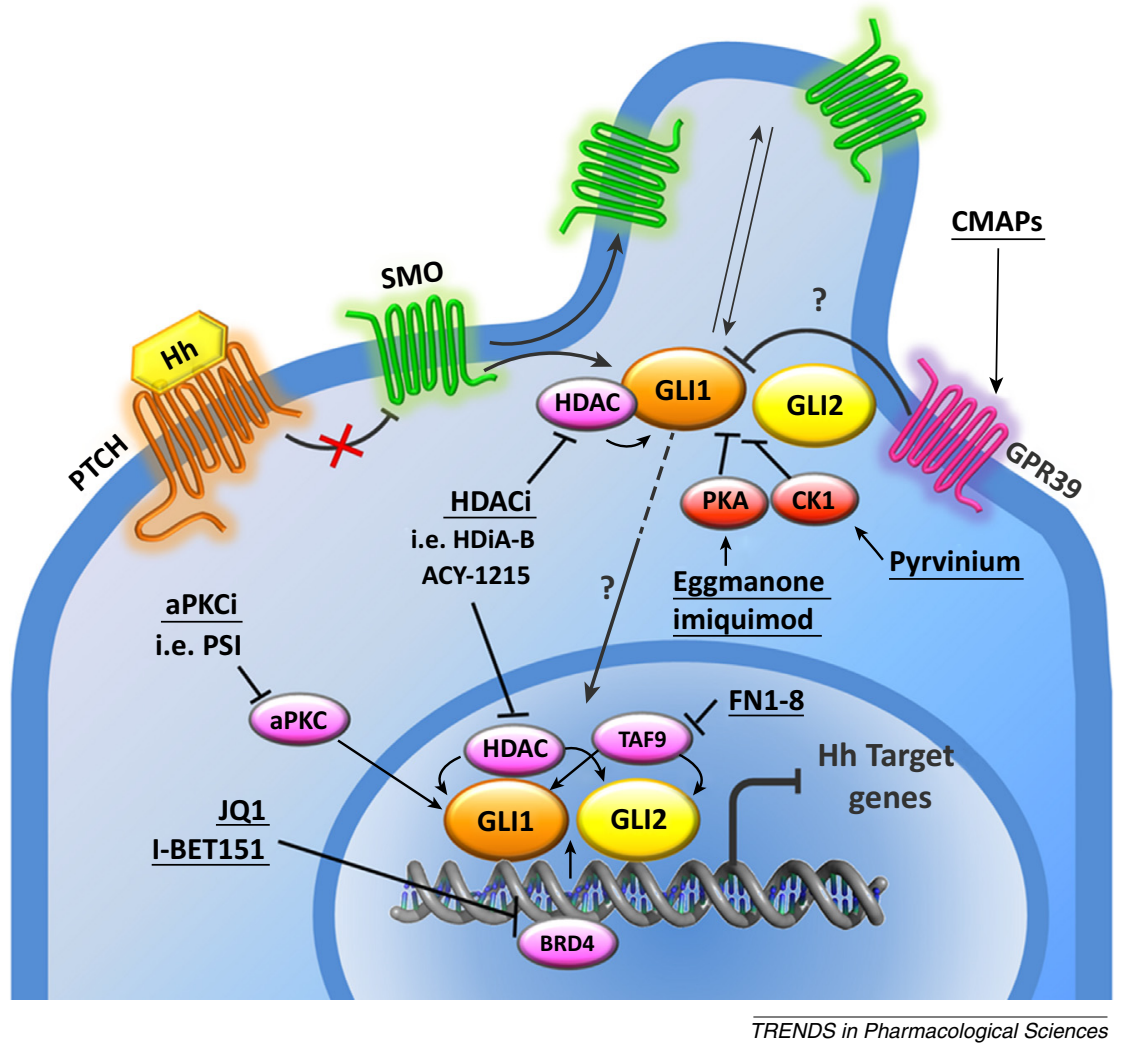

Figure 2. Mechanism of $\mathrm{Hh}$ inhibition by indirect GLI antagonists. CMAPs activate the orphan receptor GPR39, which represses GLI signaling through a not yet elucidated mechanism. Pyrvinium is an activator of CK1 kinase, while eggmanone and imiquimod are PKA activators; these molecules negatively regulate GLI transcription factors by promoting GLI2 repressor form and Gli1 degradation. HDACi, such as HDiA-B (HDAC1-2i) or ACY-1215 (HDAC6i) inhibit HDAC-mediated GLI deacetylation, which is required for GLI transcriptional activation. JQ1 and I-BET151 inhibit BRD4, which activates GLI1 and GLI2 transcription by direct occupancy of their promoters. PSI inhibits aPKC, which promotes GLI1 binding to DNA. FN1-8 affects GLI-mediated transcriptional activity by inhibiting GLI1 and GLI2/TAF9 interaction. Abbreviations: aPKC, activated protein kinase C; BRD4, bromodomain-containing protein 4; CK1, casein kinase 1; CMAPs, cyclohexyl-methyl aminopyrimidines; FN1-8,; GLI, glioma-associated oncogenes; GPR 39, G-protein-coupled receptor 39; HDACi, histone deacetylase inhibitor; Hh, Hedgehog; PKA, protein kinase A; PTCH, PATCHED; SMO, SMOOTHENED; TAF9, TBP (TATA-box binding protein)-associated factor 9.

ubiquitin-dependent degradation (Figure 2) [76]. Notably, pyrvinium attenuates $\mathrm{Hh}$-dependent $\mathrm{MB}$ cell proliferation downstream of SMO, both in vitro and in vivo (Table 1). This occurs as a consequence of its potent efficacy in downregulation of GLI1 and PTCH expression also in a context of hyperactivation of the Hh pathway, resulting from the loss of the negative regulator SUFU or overexpression of GLI proteins. Moreover, pyrvinium strongly suppressed Hh signaling induced by the oncogenic SMO mutant, which instead showed resistance to the treatment with vismodegib. Anti-SMO resistance indicates the presence of bystander co-regulatory mechanisms of the Hh pathway and suggests that their targeting may synergize with $\mathrm{Hh}$ inhibitory drugs [29]. Indeed, PI3K/AKT inhibitors revert antiSMO resistance, such as that induced by LDE225 compound [33]. Further, the ability of the mammalian target of rapamycin (mTOR)/protein-chinasi 1 p70-S6 (S6K1)-mediated phosphorylation and activation of GLI1 might explain the effect of AKT/PI3K inhibitors because of their ability to downregulate mTOR function [77].

\section{Eggmanone, imiquimod, and other SMO-independent antagonists of $G L I$}

The SMO antagonist vismodegib recently approved by the FDA for the treatment of BCC, causes frequently resistance and severe side effects. Thus, the discovery of novel clinical strategies for BCC therapy is increasingly becoming important. In this regard, new compounds, eggmanone and imiquimod, which both target Hh signaling through PKA activation, are under clinical investigation. Eggmanone (Figure 1) is a small molecule identified by an in vivo chemical genetic screening of 30000 others compounds for their ability to reproduce the Hh-null phenotype in zebrafish embryo. This molecule affects some typical structural features such as ventral tail curvature, small eyes, loss of pectoral fins, enlarged rounded somites, loss of neurocranial chondrogenesis and impaired slow muscle formation [78]. Specifically, in vitro studies show that eggmanone antagonizes Hh signaling by inhibition of phosphodiesterase (PDE)4D3, thus determining an increase of cAMP levels (Figure 2). This event leads to selective activation of PKA at the base of the primary cilium, a PKA-localization required to promote GLI processing. Imiquimod (Figure 1) is a synthetic nucleoside analog of the imidazoquinoline family, recently approved for topical treatment of small superficial BCCs. This molecule counteracts $\mathrm{Hh}$ signaling downstream of SMO by inducing PKA activity (Figure 2) with consequent GLI2/3 phosphorylation and their cleavage into repressor forms [79]. Other compounds have been identified to impinge the Hh pathway by targeting proteins and/or interactors that modulate GLI factors activity. For instance, rapamycin inhibits GLI1 functions 
<smiles>c1cc(-c2sc(-c3ccncc3)c(-c3ccncc3)c2-c2ccncc2)ccn1</smiles>

GANT58<smiles>COCCOC(=O)C1=C(C)NC2=C(C(=O)CC(c3ccccc3OC)C2)C1c1cccc(O)c1</smiles>

HPI-1<smiles>CN(C)c1ccccc1CNCCCNCc1ccccc1N(C)C</smiles>

GANT61

GANT61-diamine<smiles>COc1ccc(OC)c2c1cc1n2CC(C)(C(=O)NC2CCCCCCC2)N(CCN(C)C)C1=O</smiles><smiles>OCCNc1nc(N2CCN(c3ccccc3)CC2)nc2ccccc12</smiles><smiles>O1O[As]2O[As]3O[As]1O[As](O2)O3</smiles>

ATO<smiles>COc1cc(OC)c2c(=O)c(-c3ccc(OCC=C(C)C)c(OCC=C(C)C)c3)coc2c1</smiles>

GlaB

TRENDS in Pharmacological Sciences

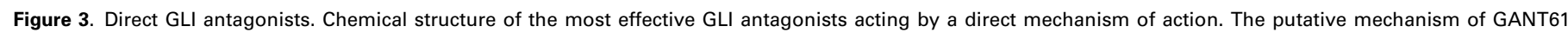

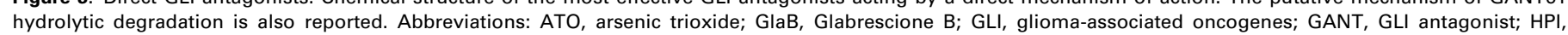
Hedgehog pathway inhibitors.

through repressing its phosphorylation and activation induced by tumor necrosis factor (TNF) $\alpha$ and mTOR/S6K1 in esophageal adenocarcinoma cells [77]. FN1-8, a synthetic small molecule comprising a pyrazoline structure (Figure 1), strongly reduces GLI-mediated transcriptional activity by disrupting the interaction of both GLI1 and GLI2 with TBP (TATA box-binding protein)-associated factor (TAF)9, a transcription coactivator (Figure 2). Noteworthy, FN1-8 was able to suppress the proliferation of lung cancer cells in vitro and in vivo and to inhibit the cell growth of numerous cancer cells that express high GLI levels, including prostate, pancreatic, colon cancer, and glioma [80].

\section{Direct GLI antagonists}

GLI antagonist 61 (GANT61)

In 2007, a cellular screen for small molecule inhibitors of GLI1-mediated transcription led to the identification of GANT58 and GANT61 (Figure 3) as the first GLI antagonists [81]. GANT61 proved to counteract GLI-mediated tumor growth more efficiently than GANT58, becoming therefore the lead candidate for further investigations and the most valuable tool for pharmacological Hh pathway inhibition. Although its mechanism of action has not been elucidated and no records of preclinical or clinical studies are available, GANT61 is the reference GLI1/GLI2 antagonist in many biological and drug design studies. GANT61 inhibits Hh signaling by impairing GLI1- and GLI2-mediated transcription in vitro and in vivo, affecting the GLI1/ DNA interaction only in living cells probably by inducing post-translational modifications of GLI1 (Figure 4) [81]. Moreover, GANT61 is poorly stable at physiological conditions, and quickly hydrolyzes into a benzaldehyde species (inactive against $\mathrm{Hh}$ signaling) and a diamine derivative (Figure 3), which has showed the same efficacy as GANT61 in inhibiting GLI-mediated transcription [82].

GANT61 has been successfully used to inhibit the HhGLI1 axis at the GLI1 and/or GLI2 level in several cancer cells, CSCs, and tumor animal models, such as pancreatic, [83] prostate and lung cancer [81,84], colon [85] and hepatocellular carcinoma (Table 1) [86], as well as against tumors for which the role of GLI transcription was not elucidated before, including ovarian cancer [87], esophageal adenocarcinoma [88], and melanoma CSCs [89], emphasizing the therapeutic potential of GANT61. Finally, GANT61 has been used to monitor the relationship between specific GLI targeting and autophagy. Indeed, GANT61 has been found to induce cancer suppressing autophagic processes in PDAC and hepatocellular carcinoma cells, whereas prosurvival autophagy has been observed in neuroblastoma cells [86,90,91].

\section{Arsenic trioxide (ATO)}

ATO (Figure 3) is an FDA-approved drug for second-line treatment of acute promyelocytic leukemia that functions by binding to cysteine residues of proteins involved in MAPK, nuclear factor $\kappa$-light-chain-enhancer of activated B cells (NF-к,B), c-Jun N-terminal kinases (JNK) and other pathways. In 2010, Kim and collaborators observed that ATO antagonizes the Hh pathway in vitro and in vivo by 


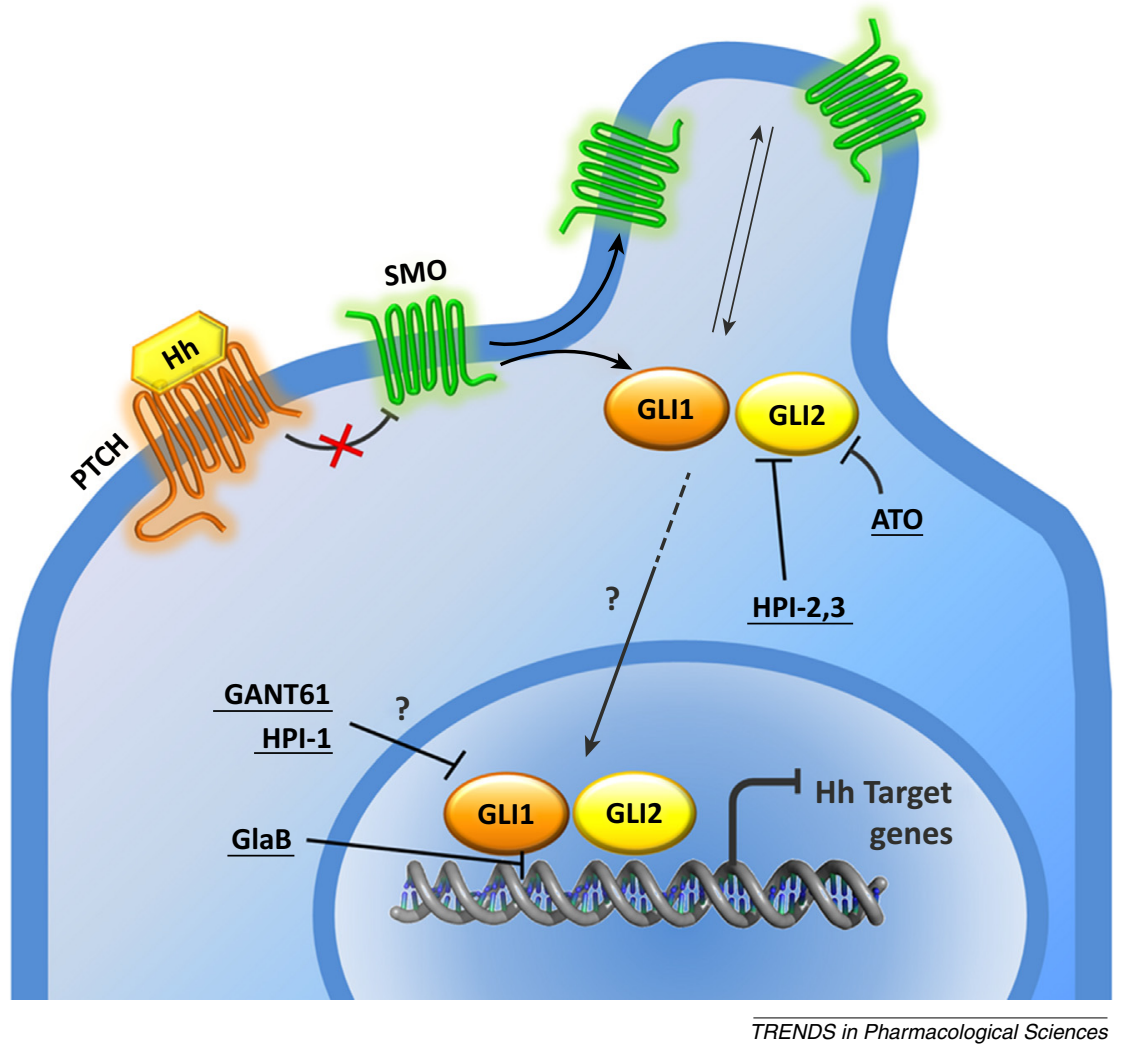

Figure 4. Mechanism of $\mathrm{Hh}$ inhibition by direct GLI antagonists. GANT61 inhibits GLI-mediated transcription by an unclear mechanism (most likely by inducing posttranslational modifications of GLI). ATO affects ciliary accumulation of GLI2 and enhances its degradation after longer treatment regimens. HPI-1 has been observed to

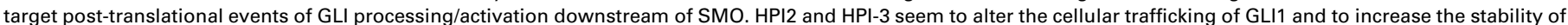

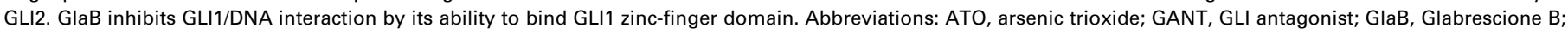
GLI, glioma-associated oncogenes; Hh, Hedgehog; HPI, Hedgehog pathway inhibitors; PTCH, PATCHED; SMO, SMOOTHENED.

directly interfering with GLI transcription factors (Table 1) [92]. ATO has been observed to inhibit ciliary accumulation of GLI2 in the short term, and to enhance GLI2 degradation after a prolonged incubation time in MB cells (Figure 4). In 2011, Beauchamp et al. showed that ATO inhibits $\mathrm{Hh}$ signaling by interacting directly with GLI1, without altering GLI1/DNA interaction or modifying GLI1 cellular trafficking and stability [93]. Although these works have proposed rather different mechanisms, they have clearly indicated that ATO, used alone or in combination with other anticancer drugs, may represent a valuable therapeutic option to treat Hh-dependent tumors, particularly those harboring drug-resistant SMO mutations [92-94]. Accordingly, itraconazole, an FDA-approved triazole antifungal agent, operates on the Hh signaling at the level of SMO at a different site than cyclopamine. Single and combined therapy with itraconazole and ATO inhibits cellular growth of MB and BCC in vitro and in vivo, both in wild type and in drugresistant $\mathrm{SMO}^{\mathrm{D} 477 \mathrm{G}}$ mice. In fact, the problem of resistance establishment to cyclopamine or cyclopamine mimics, due to some human SMO point mutations (such as $\mathrm{D} 473 \mathrm{H}$ and E518K), is becoming even more prevalent. These characteristics strongly encourage the synergic therapy with itraconazole and ATO for the treatment of de novo Hh-dependent tumors or those with acquired resistance to cyclopamine [94]. Following these studies, the efficacy of ATO in targeting GLI transcription factors has been investigated in MB [93-95], pleural mesothelioma [96], malignant rhabdoid tumors [97], osteosarcoma [98], and many others cancer types. Furthermore, growing evidence points to a role for ATO as a modulator of the proliferation and differentiation of cancer cells progenitors by impacting on Hh or Notch signaling [99]. It is worth mentioning that a pilot clinical trial study to monitor the efficacy of ATO in treating patients with BCC started in late 2013 (https://clinicaltrials.gov/ show/NCT01791894).

\section{Hedgehog pathway inhibitors (HPIs)}

A cell-based high-throughput screening performed by Hyman and coworkers led to the identification of some chemically unrelated small molecules (namely, HPI-1, HPI-2, HPI-3, and HPI-4) (Figure 3), which inhibit the Hh pathway downstream of SMO by directly antagonizing GLI (Table 1) [100]. Collectively, HPIs have demonstrated multiple and different mechanisms of action (Figure 4). HPI-1 has been observed to target post-translational events of GLI processing/activation downstream of SMO and has been shown to inhibit the growth of hepatocellular carcinoma and MB cells expressing drug-resistant SMO, especially when encapsulated in nanoparticles (Table 1) [101]. In contrast, HPI-2 and HPI-3 have been associated with an altered trafficking of GLI1 and with increased stability of GLI2, which in turn impairs the conversion of full-length GLI2 to a transcriptional activator. Finally, HPI-4 has been found to perturb ciliogenesis by an unclear mechanism, and to decrease human chondrosarcoma cell proliferation, invasion, and migration [102]. Only HPI-1 and HPI-4 have been shown to inhibit the proliferation of 
(A)

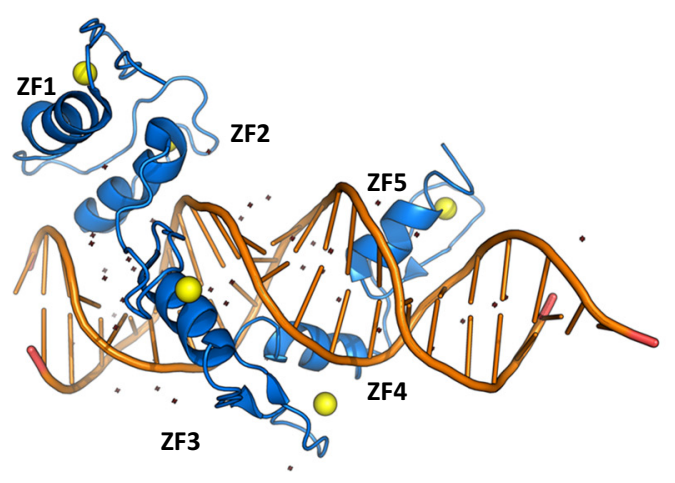

(B)

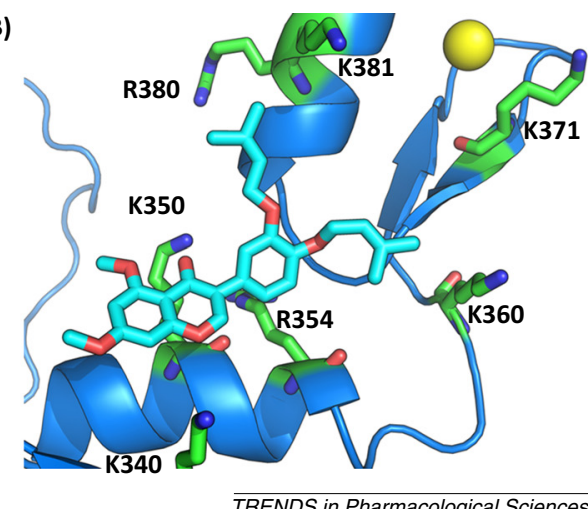

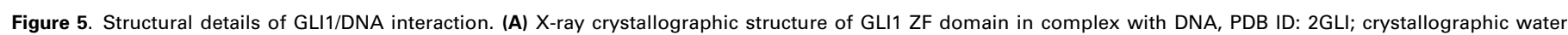
molecules are shown as red dots. (B) Details of the predicted binding pose of GlaB (cyan stick) within ZF4 and ZF5. Key residues for GLI1 transcriptional functions are shown as green sticks. Metal ions are shown as yellow spheres. Abbreviations: GlaB, Glabrescione B; GLI, glioma-associated oncogenes; ZF, zinc finger.

cerebellar granule neuron precursors. The multiple effects of HPIs on Hh signaling have highlighted the complexity of Hh/GLI regulation and have offered different opportunities for pharmacological modulation of aberrant Hh signaling even though their efficacy in vivo has not been evaluated yet.

\section{Glabrescione $B$ (GlaB)}

Natural products have historically had a significant impact in Hh modulation (i.e., cyclopamine). Among the various chemical classes of natural products, isoflavones have displayed a noticeable pharmacophoric preference for $\mathrm{Hh}$ targets, as underlined by genistein and its derivatives in differentiated pancreatic cancer cells and CSCs [103,104]. Recently, our own research group has established a multidisciplinary drug discovery program focusing on the identification of natural products as direct GLI1 antagonists. Starting from the crystallographic structure of the GLI1-ZF domain in a complex with DNA [105], we have performed a mixed computational and experimental structure-based study to identify K350, R354, R380, and K381 residues as the strongest hot spots for GLI1/DNA interaction and transcriptional functions (Figure 5) [106]. This information was subsequently used to discover pharmacological agents able to interfere with this basic process. By virtual screening of a natural compounds library against these hot spots and monitoring Hh inhibition at GLI1 level, we have identified GlaB (Figures 3 and 5B, Table 1), an isoflavone naturally occurring in Derris glabrescens, as a potent Hh inhibitor acting downstream of SMO. NMR and (EMSA) Electrophoretic Mobility Shift Assay experiments have confirmed that GlaB binds to GLI1 in correspondence of ZF4 and ZF5 affecting GLI1/DNA interaction and also emphasizing the role of K350 and K340 in this event [106] (Figures 4 and 5). GlaB has proved to inhibit Hh signaling in multiple cancer cells and CSCs, as well as the in vivo $\mathrm{MB}$ and BCC tumor growth in orthotopic xenograft mice and in allograft mice models, respectively (Table 1) [106]. Our work has raised the importance to investigate the mechanism of GLI1-mediated transcription, and the identification of the structural requirements of GLI1/DNA interaction have highlighted their relevance for pharmacological interference of GLI1 signaling by direct GLI1 antagonists.

\section{Concluding remarks}

A critical goal in Hh-dependent tumor biology is the discovery of novel small molecules blocking the pathway at a downstream level. The rationale for the development of these pharmacological agents is based on (i) the heterogeneity of molecular defects sustaining the pathway activation; and (ii) the need to overcome the resistance to clinically available SMO antagonists.

Targeting GLI effectors represents a promising therapeutic strategy for cancer treatment. This is particularly relevant for certain tumors, such as $\mathrm{MB}$, since, although classified into four distinct molecular groups (Hh- or Wnttype and 3rd or 4th), common signals, such as placental growth factor (PIGF)/neuropilin axis, responsible of GLI1 upregulation, are shared by all subtypes and are important for tumor growth [107].

Transcription factors are generally considered as challenging targets in drug discovery for many different reasons, including the lack of deep hydrophobic pockets that accommodate small molecules and the highly charged surface. Nevertheless, big pharmaceutical companies are currently running Phase I clinical trials with small molecules targeting transcription factors such as Notch (BristolMyers Squibb and Ely Lilly) and p53 (Roche and Sanofi) [108], although no drugs have been approved yet by the FDA. Beside these targets, cMYC, NF-кB, signal transducer and activator of transcription (STAT)3, STAT5, AP1, hypoxia-inducible factor (HIF1), and GLI1 are among the most promising transcription factors for the development of new anticancer drugs. GLI functions are finely tuned by a number of molecular interactions and postsynthetic modifications (i.e., GLI1 phosphorylation, gene copy number amplification, BRD4-driven epigenetic activation, deubiquitylation, deacetylation, or activation by $\mathrm{aPKC}, / \mathrm{k}$ or $\mathrm{p} 70 \mathrm{~S} 6 \mathrm{~K}$ or RAS/ERK) that, if dysregulated, are responsible for the resistance to anti-SMO drugs frequently observed in Hh-driven tumor initiation, progression and relapse [16,26, 109]. Interestingly, GLI-processing and activating posttranslational events are pharmacologically targetable, even though by means of combination therapies. For this reason, the challenge in this field is the understanding of the molecular mechanisms that regulate GLI-mediated transcription. In this regard, the recently reported identification of the structural requirements of GLI1/DNA interaction, 
stands as a promising tool for discovering small molecules capable of inhibiting Hh pathway by directly targeting GLI [106]. The discovery of the natural compound, GlaB, able to impair Hh oncogenic activity by inhibiting GLI1/DNA interaction, provides a proof-of-principle for the therapeutic relevance of such an approach, focused on the unique downstream GLI1 transcriptional effector rather than on multiple upstream oncogenic deregulated signals. Since the GLI proteins share highly conserved sequence of their $\mathrm{ZF}$ domain, targeting GLI1/DNA interaction could also interfere with GLI3/DNA binding, counteracting the GLI3 repressor function and resulting in severe side effects. However, the use of $\mathrm{Hh}$ antagonists is thought in a context of aberrant $\mathrm{Hh}$ pathway activation, where GLI repressor forms are absent, so bypassing the possibility of nonspecific effects on negative regulatory activity of GLI factors. Therefore, drugs specifically designed to modulate GLI/DNA interaction would provide valuable insights for developing and optimizing GLI antagonists, which would promise a more effective treatment of Hh-dependent tumors.

\section{Acknowledgments}

We apologize to those whose work was left out of this review owing to space limitations. We thank I. Screpanti for a critical reading of this manuscript. This work was supported by AIRC (Associazione Italiana Ricerca Cancro) grant \#IG14723, the Ministry of University and Research (PRIN project), IIT (Italian Institute of Technology), and Pasteur Institute/Cenci Bolognetti Foundation. This review article is dedicated to the memory of Alberto Gulino an outstanding scientist, our dear friend and mentor.

\section{References}

1 Varjosalo, M. and Taipale, J. (2008) Hedgehog: functions and mechanisms. Genes Dev. 22, 2454-2472

2 Ryan, K.E. and Chiang, C. (2012) Hedgehog secretion and signal transduction in vertebrates. J. Biol. Chem. 287, 17905-17913

3 Benazet, J.D. and Zeller, R. (2009) Vertebrate limb development: moving from classical morphogen gradients to an integrated 4-dimensional patterning system. Cold Spring Harb. Perspect. Biol. 1, a001339

4 Gill, P.S. and Rosenblum, N.D. (2006) Control of murine kidney development by sonic hedgehog and its GLI effectors. Cell Cycle 5, 1426-1430

5 Kugler, M.C. et al. (2015) Sonic hedgehog signaling in the lung. From development to disease. Am. J. Respir. Cell Mol. Biol. 52, 1-13

6 Hynes, A.M. et al. (2014) Murine Joubert syndrome reveals Hedgehog signaling defects as a potential therapeutic target for nephronophthisis. Proc. Natl. Acad. Sci. U.S.A. 111, 9893-9898

7 Lipinski, R.J. et al. (2014) Characterization of subtle brain abnormalities in a mouse model of Hedgehog pathway antagonistinduced cleft lip and palate. PLoS ONE 9, e102603

8 Bian, Y.H. et al. (2007) Sonic hedgehog-Gli1 pathway in colorectal adenocarcinomas. World J. Gastroenterol. 13, 1659-1665

9 Gerber, A.N. et al. (2007) The hedgehog regulated oncogenes Gli1 and Gli2 block myoblast differentiation by inhibiting MyoD-mediated transcriptional activation. Oncogene 26, 1122-1136

10 Ruiz i Altaba, A. et al. (2002) Gli and hedgehog in cancer: tumours, embryos and stem cells. Nat. Rev. Cancer 2, 361-372

11 Sanchez, P. et al. (2004) Inhibition of prostate cancer proliferation by interference with SONIC HEDGEHOG-GLI1 signaling. Proc. Natl. Acad. Sci. U.S.A. 101, 12561-12566

12 Gailani, M.R. et al. (1996) The role of the human homologue of Drosophila patched in sporadic basal cell carcinomas. Nat. Genet. 14, 78-81

13 Taylor, M.D. et al. (2002) Mutations in SUFU predispose to medulloblastoma. Nat. Genet. 31, 306-310

14 Xie, J. et al. (1998) Activating Smoothened mutations in sporadic basal-cell carcinoma. Nature 391, 90-92
15 Kinzler, K.W. et al. (1987) Identification of an amplified, highly expressed gene in a human glioma. Science 236, 70-73

16 Aberger, F. and Ruiz, I.A.A. (2014) Context-dependent signal integration by the GLI code: the oncogenic load, pathways, modifiers and implications for cancer therapy. Semin. Cell Dev. Biol. 33, 93-104

17 Coni, S. et al. (2013) Control of stem cells and cancer stem cells by Hedgehog signaling: pharmacologic clues from pathway dissection. Biochem. Pharmacol. 85, 623-628

18 Di Marcotullio, L. et al. (2011) Protected from the inside: endogenous histone deacetylase inhibitors and the road to cancer. Biochim. Biophys. Acta 1815, 241-252

19 Po, A. et al. (2010) Hedgehog controls neural stem cells through p53-independent regulation of Nanog. EMBO J. 29, 2646-2658

20 Varnat, F. et al. (2009) Human colon cancer epithelial cells harbour active HEDGEHOG-GLI signalling that is essential for tumour growth, recurrence, metastasis and stem cell survival and expansion. EMBO Mol. Med. 1, 338-351

21 Chen, J.K. et al. (2002) Inhibition of Hedgehog signaling by direct binding of cyclopamine to Smoothened. Genes Dev. 16, 2743-2748

22 Lipinski, R.J. et al. (2008) Dose- and route-dependent teratogenicity, toxicity, and pharmacokinetic profiles of the hedgehog signaling antagonist cyclopamine in the mouse. Toxicol. Sci. 104, 189-197

23 Hadden, M.K. (2013) Hedgehog pathway inhibitors: a patent review (2009 - present). Expert Opin. Ther. Pat. 23, 345-361

24 Manetti, F. et al. (2014) Structure-activity relationships and mechanism of action of small molecule smoothened modulators discovered by high-throughput screening and rational design. Top. Med. Chem. Published online October 25, 2014. http://dx.doi.org/ 10.1007/7355_2014_61

25 Ruat, M. et al. (2014) Targeting of Smoothened for therapeutic gain. Trends Pharmacol. Sci. 35, 237-246

26 Amakye, D. et al. (2013) Unraveling the therapeutic potential of the Hedgehog pathway in cancer. Nat. Med. 19, 1410-1422

$27 \mathrm{Ng}$, J.M. and Curran, T. (2011) The Hedgehog's tale: developing strategies for targeting cancer. Nat. Rev. Cancer 11, 493-501

28 Dlugosz, A. et al. (2012) Vismodegib. Nat. Rev. Drug Discov. 11, 437-438

29 Yauch, R.L. et al. (2009) Smoothened mutation confers resistance to a Hedgehog pathway inhibitor in medulloblastoma. Science 326, $572-574$

30 Jimeno, A. et al. (2013) Phase I study of the Hedgehog pathway inhibitor IPI-926 in adult patients with solid tumors. Clin. Cancer Res. 19, 2766-2774

31 Atwood, S.X. et al. (2015) Smoothened variants explain the majority of drug resistance in basal cell carcinoma. Cancer Cell 27, 342-353

32 Sharpe, H.J. et al. (2015) Genomic analysis of smoothened inhibitor resistance in basal cell carcinoma. Cancer Cell 27, 327-341

33 Buonamici, S. et al. (2010) Interfering with resistance to smoothened antagonists by inhibition of the PI3K pathway in medulloblastoma. Sci. Transl. Med. 2, 51ra70

34 Lee, M.J. et al. (2012) Hedgehog pathway inhibitor saridegib (IPI-926) increases lifespan in a mouse medulloblastoma model. Proc. Natl. Acad. Sci. U.S.A. 109, 7859-7864

35 Metcalfe, C. and de Sauvage, F.J. (2011) Hedgehog fights back: mechanisms of acquired resistance against Smoothened antagonists. Cancer Res. 71, 5057-5061

36 Rudin, C.M. et al. (2009) Treatment of medulloblastoma with hedgehog pathway inhibitor GDC-0449. N. Engl. J. Med. 361, $1173-1178$

37 Tang, J.Y. et al. (2012) Inhibiting the hedgehog pathway in patients with the basal-cell nevus syndrome. N. Engl. J. Med. 366, 2180-2188

38 Teperino, R. et al. (2012) Hedgehog partial agonism drives Warburglike metabolism in muscle and brown fat. Cell 151, 414-426

39 Huntzicker, E.G. et al. (2006) Dual degradation signals control Gli protein stability and tumor formation. Genes Dev. 20, 276-281

40 Di Marcotullio, L. et al. (2007) Multiple ubiquitin-dependent processing pathways regulate hedgehog/gli signaling: implications for cell development and tumorigenesis. Cell Cycle 6, 390-393

41 Gulino, A. et al. (2012) Hedgehog/Gli control by ubiquitination/ acetylation interplay. Vitam. Horm. 88, 211-227

42 Katoh, Y. and Katoh, M. (2009) Hedgehog target genes: mechanisms of carcinogenesis induced by aberrant hedgehog signaling activation. Curr. Mol. Med. 9, 873-886 
43 Di Marcotullio, L. et al. (2006) Numb is a suppressor of Hedgehog signalling and targets Gli1 for Itch-dependent ubiquitination. Nat. Cell Biol. 8, 1415-1423

44 Di Marcotullio, L. et al. (2011) Numb activates the E3 ligase Itch to control Gli1 function through a novel degradation signal. Oncogene $30,65-76$

45 Canettieri, G. et al. (2010) Histone deacetylase and Cullin3REN(KCTD11) ubiquitin ligase interplay regulates Hedgehog signalling through Gli acetylation. Nat. Cell Biol. 12, 132-142

46 De Smaele, E. et al. (2011) Identification and characterization of KCASH2 and KCASH3, 2 novel Cullin3 adaptors suppressing histone deacetylase and Hedgehog activity in medulloblastoma. Neoplasia 13, 374-385

47 Stecca, B. and Ruiz, I.A.A. (2010) Context-dependent regulation of the GLI code in cancer by HEDGEHOG and non-HEDGEHOG signals. J. Mol. Cell Biol. 2, 84-95

48 Clement, V.et al. (2007) HEDGEHOG-GLI1 signaling regulates human glioma growth, cancer stem cell self-renewal, and tumorigenicity. Curr. Biol. 17, 165-172

49 Hatta, N. et al. (2005) Molecular diagnosis of basal cell carcinoma and other basaloid cell neoplasms of the skin by the quantification of Gli1 transcript levels. J. Cutan. Pathol. 32, 131-136

50 Snijders, A.M. et al. (2009) Stromal control of oncogenic traits expressed in response to the overexpression of GLI2, a pleiotropic oncogene. Oncogene 28, 625-637

51 Alexaki, V.I. et al. (2010) GLI2-mediated melanoma invasion and metastasis. J. Natl. Cancer Inst. 102, 1148-1159

52 Thiyagarajan, S. et al. (2007) Role of GLI2 transcription factor in growth and tumorigenicity of prostate cells. Cancer Res. 67, 1064210646

53 Sheng, H. et al. (2002) Dissecting the oncogenic potential of Gli2: deletion of an $\mathrm{NH}(2)$-terminal fragment alters skin tumor phenotype. Cancer Res. 62, 5308-5316

$54 \mathrm{Kim}, \mathrm{Y}$. et al. (2007) Selective down-regulation of glioma-associated oncogene 2 inhibits the proliferation of hepatocellular carcinoma cells. Cancer Res. 67, 3583-3593

55 Pandolfi, S. and Stecca, B. (2015) Cooperative integration between HEDGEHOG-GLI signalling and other oncogenic pathways: implications for cancer therapy. Expert Rev. Mol. Med. 17, e5

56 Mimeault, M. and Batra, S.K. (2010) Frequent deregulations in the hedgehog signaling network and cross-talks with the epidermal growth factor receptor pathway involved in cancer progression and targeted therapies. Pharmacol. Rev. 62, 497-524

57 Nolan-Stevaux, O. et al. (2009) GLI1 is regulated through Smoothened-independent mechanisms in neoplastic pancreatic ducts and mediates PDAC cell survival and transformation. Genes Dev. 23, 24-36

58 Schnidar, H. et al. (2009) Epidermal growth factor receptor signaling synergizes with Hedgehog/GLI in oncogenic transformation via activation of the MEK/ERK/JUN pathway. Cancer Res. 69, 1284-1292

59 Xie, J. et al. (2001) A role of PDGFRalpha in basal cell carcinoma proliferation. Proc. Natl. Acad. Sci. U.S.A. 98, 9255-9259

60 Beauchamp, E. et al. (2009) GLI1 is a direct transcriptional target of EWS-FLI1 oncoprotein. J. Biol. Chem. 284, 9074-9082

61 Riobo, N.A. et al. (2006) Phosphoinositide 3-kinase and Akt are essential for Sonic Hedgehog signaling. Proc. Natl. Acad. Sci. U.S.A. 103, 4505-4510

62 Atwood, S.X. et al. (2013) GLI activation by atypical protein kinase C iota/lambda regulates the growth of basal cell carcinomas. Nature 494, 484-488

63 Bassilana, F. et al. (2014) Target identification for a Hedgehog pathway inhibitor reveals the receptor GPR39. Nat. Chem. Biol. $10,343-349$

64 Filippakopoulos, P. et al. (2010) Selective inhibition of BET bromodomains. Nature 468, 1067-1073

65 Yang, Z. et al. (2005) Recruitment of P-TEFb for stimulation of transcriptional elongation by the bromodomain protein Brd4. Mol. Cell 19, 535-545

66 Long, J. et al. (2014) The BET bromodomain inhibitor I-BET151 acts downstream of smoothened protein to abrogate the growth of hedgehog protein-driven cancers. J. Biol. Chem. 289, 35494-35502
67 Wang, Y.H. et al. (2015) BRD4 promotes pancreatic ductal adenocarcinoma cell proliferation and enhances gemcitabine resistance. Oncol. Rep. 33, 1699-1706

68 Coni, S. et al. (2013) Gli2 acetylation at lysine 757 regulates hedgehogdependent transcriptional output by preventing its promoter occupancy. PLoS ONE 8, e65718

69 Milde, T. et al. (2010) HDAC5 and HDAC9 in medulloblastoma: novel markers for risk stratification and role in tumor cell growth. Clin. Cancer Res. 16, 3240-3252

70 Dhanyamraju, P.K. et al. (2014) Histone deacetylase 6 represents a novel drug target in the oncogenic Hedgehog signaling pathway. Mol. Cancer Ther. 14, 727-739

71 Mottamal, M. et al. (2015) Histone deacetylase inhibitors in clinical studies as templates for new anticancer agents. Molecules 20, 38983941

72 Slingerland, M. et al. (2014) Histone deacetylase inhibitors: an overview of the clinical studies in solid tumors. Anticancer. Drugs $25,140-149$

73 Spiller, S.E. et al. (2006) Suberoylanilide hydroxamic acid is effective in preclinical studies of medulloblastoma. J. Neurooncol. 79, 259-270

$74 \mathrm{Li}$, B. et al. (2014) Pyrvinium attenuates Hedgehog signaling downstream of smoothened. Cancer Res. 74, 4811-4821

$75 \mathrm{Li}$, B. et al. (2014) Repurposing the FDA-approved pinworm drug pyrvinium as a novel chemotherapeutic agent for intestinal polyposis. PLoS ONE 9, e101969

76 Ruiz i Altaba, A. et al. (2007) The Gli code: an information nexus regulating cell fate, stemness and cancer. Trends Cell Biol. 17, 438-447

77 Wang, Y. et al. (2012) The crosstalk of mTOR/S6K1 and Hedgehog pathways. Cancer Cell 21, 374-387

78 Williams, C.H. et al. (2015) An in vivo chemical genetic screen identifies phosphodiesterase 4 as a pharmacological target for hedgehog signaling inhibition. Cell Rep. 11, 43-50

79 Wolff, F. et al. (2013) Imiquimod directly inhibits Hedgehog signalling by stimulating adenosine receptor/protein kinase A-mediated GLI phosphorylation. Oncogene $32,5574-5581$

80 Bosco-Clement, G. et al. (2014) Targeting Gli transcription activation by small molecule suppresses tumor growth. Oncogene 33, 2087-2097

81 Lauth, M. et al. (2007) Inhibition of GLI-mediated transcription and tumor cell growth by small-molecule antagonists. Proc. Natl. Acad. Sci. U.S.A. 104, 8455-8460

82 Lauth, M. et al. (2010) Antipsychotic drugs regulate hedgehog signaling by modulation of 7-dehydrocholesterol reductase levels. Mol. Pharmacol. 78, 486-496

$83 \mathrm{Fu}$, J. et al. (2013) GANT-61 inhibits pancreatic cancer stem cell growth in vitro and in NOD/SCID/IL2R gamma null mice xenograft. Cancer Lett. 330, 22-32

84 Huang, L. et al. (2014) Hedgehog-GLI signaling inhibition suppresses tumor growth in squamous lung cancer. Clin. Cancer Res. 20, 1566-1575

85 Mazumdar, T. et al. (2011) Blocking Hedgehog survival signaling at the level of the GLI genes induces DNA damage and extensive cell death in human colon carcinoma cells. Cancer Res. 71, 5904-5914

86 Wang, Y. et al. (2013) Hedgehog signaling pathway regulates autophagy in human hepatocellular carcinoma cells. Hepatology 58 , 995-1010

87 Chen, Q. et al. (2014) Down-regulation of Gli transcription factor leads to the inhibition of migration and invasion of ovarian cancer cells via integrin beta4-mediated FAK signaling. PLoS ONE 9, e88386

88 Kebenko, M. et al. (2015) ErbB2 signaling activates the Hedgehog pathway via PI3K-Akt in human esophageal adenocarcinoma: identification of novel targets for concerted therapy concepts. Cell. Signal. 27, 373-381

89 Santini, R. et al. (2012) Hedgehog-GLI signaling drives self-renewal and tumorigenicity of human melanoma-initiating cells. Stem Cells 30, 1808-1818

90 Wang, J. et al. (2014) Inhibition of autophagy potentiates the efficacy of Gli inhibitor GANT-61 in MYCN-amplified neuroblastoma cells. BMC Cancer 14, 768

$91 \mathrm{Xu}, \mathrm{Y}$. et al. (2014) Inhibition of the Hedgehog pathway induces autophagy in pancreatic ductal adenocarcinoma cells. Oncol. Rep. 31, 707-712

$92 \mathrm{Kim}$, J. et al. (2010) Arsenic antagonizes the Hedgehog pathway by preventing ciliary accumulation and reducing stability of the Gli2 
transcriptional effector. Proc. Natl. Acad. Sci. U.S.A. 107, 1343213437

93 Beauchamp, E.M. et al. (2011) Arsenic trioxide inhibits human cancer cell growth and tumor development in mice by blocking Hedgehog/ GLI pathway. J. Clin. Invest. 121, 148-160

$94 \mathrm{Kim}$, J. et al. (2013) Itraconazole and arsenic trioxide inhibit Hedgehog pathway activation and tumor growth associated with acquired resistance to smoothened antagonists. Cancer Cell 23, 23-34

95 Di Magno, L. et al. (2014) Druggable glycolytic requirement for Hedgehog-dependent neuronal and medulloblastoma growth. Cell Cycle 13, 3404-3413

96 You, M. et al. (2014) Targeting of the Hedgehog signal transduction pathway suppresses survival of malignant pleural mesothelioma cells in vitro. J. Thorac. Cardiovasc. Surg. 147, 508-516

97 Kerl, K. et al. (2014) Arsenic trioxide inhibits tumor cell growth in malignant rhabdoid tumors in vitro and in vivo by targeting overexpressed Gli1. Int. J. Cancer 135, 989-995

98 Nakamura, S. et al. (2013) Arsenic trioxide prevents osteosarcoma growth by inhibition of GLI transcription via DNA damage accumulation. PLoS ONE 8, e69466

99 Ding, D. et al. (2014) Arsenic trioxide inhibits Hedgehog, Notch and stem cell properties in glioblastoma neurospheres. Acta Neuropathol. Commun. 2, 31

100 Hyman, J.M. et al. (2009) Small-molecule inhibitors reveal multiple strategies for Hedgehog pathway blockade. Proc. Natl. Acad. Sci. U.S.A. 106, 14132-14137
$101 \mathrm{Xu}$, Y. et al. (2012) Polymeric nanoparticle-encapsulated hedgehog pathway inhibitor HPI-1 (NanoHHI) inhibits systemic metastases in an orthotopic model of human hepatocellular carcinoma. Clin. Cancer Res. 18, 1291-1302

102 Xiang, W. et al. (2014) Hedgehog pathway inhibitor-4 suppresses malignant properties of chondrosarcoma cells by disturbing tumor ciliogenesis. Oncol. Rep. 32, 1622-1630

103 Slusarz, A. et al. (2010) Common botanical compounds inhibit the hedgehog signaling pathway in prostate cancer. Cancer Res. 70, 3382-3390

104 Zhang, L. et al. (2012) Genistein inhibits the stemness properties of prostate cancer cells through targeting Hedgehog-Gli1 pathway. Cancer Lett. 323, 48-57

105 Pavletich, N.P. and Pabo, C.O. (1993) Crystal structure of a five-finger GLI-DNA complex: new perspectives on zinc fingers. Science 261, 1701-1707

106 Infante, P. et al. (2015) Gli1/DNA interaction is a druggable target for Hedgehog-dependent tumors. EMBO J. 34, 200-217

107 Snuderl, M. et al. (2013) Targeting placental growth factor/neuropilin 1 pathway inhibits growth and spread of medulloblastoma. Cell 152, 1065-1076

108 Roemer, K. (2012) Notch and the p53 clan of transcription factors. Adv. Exp. Med. Biol. 727, 223-240

109 Tang, Y. et al. (2014) Epigenetic targeting of Hedgehog pathway transcriptional output through BET bromodomain inhibition. Nat. Med. 20, 732-740 